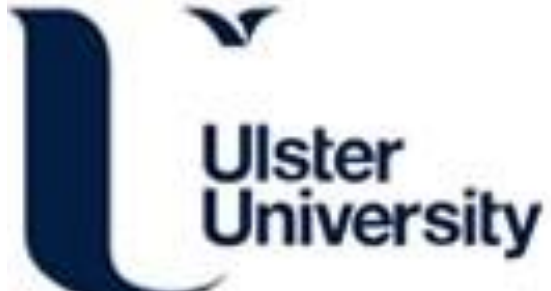

\section{A review of the impact of exercise on treatment related fatigue among patients receiving adjuvant radiotherapy for breast cancer.}

McNally, F., Shepherd, P., \& Flood, T. (2019). A review of the impact of exercise on treatment related fatigue among patients receiving adjuvant radiotherapy for breast cancer. Journal of Radiotherapy in Practice, 18(3), 295-300. https://doi.org/10.1017/S1460396918000754

Link to publication record in Ulster University Research Portal

Published in:

Journal of Radiotherapy in Practice

Publication Status:

Published (in print/issue): 01/09/2019

DOI:

10.1017/S1460396918000754

\section{Document Version}

Author Accepted version

\section{General rights}

Copyright for the publications made accessible via Ulster University's Research Portal is retained by the author(s) and / or other copyright owners and it is a condition of accessing these publications that users recognise and abide by the legal requirements associated with these rights.

\section{Take down policy}

The Research Portal is Ulster University's institutional repository that provides access to Ulster's research outputs. Every effort has been made to ensure that content in the Research Portal does not infringe any person's rights, or applicable UK laws. If you discover content in the Research Portal that you believe breaches copyright or violates any law, please contact pure-support@ulster.ac.uk. 


\section{$\underline{\text { Title }}$}

A review of the impact of exercise on treatment related fatigue among patients receiving adjuvant radiotherapy for breast cancer.

Fiona McNally BSc (Hons), Western Health and Social Care Trust.

Paul Shepherd OBE MSc, Ulster University.

Terri Flood MSc, Ulster University.

Corresponding Author: Paul Shepherd OBE, School of Health Sciences, Ulster University, Newtownabbey, County Antrim, BT37 0QB, UNITED KINGDOM

Email:PH.Shepherd@ulster.ac.uk

Telephone: +44 (0)2890366233

\section{Keywords}

Breast cancer; Adjuvant Radiotherapy; Chemotherapy; Fatigue; Exercise. 


\section{Abstract}

Purpose

To evaluate the use of exercise in managing fatigue in breast cancer patients undergoing adjuvant radiotherapy. To explore the effectiveness of different exercise practices and explore how optimum management of fatigue might be achieved.

Method

A CINAHL database search of literature was undertaken and publications screened for retrieval with twenty-four qualifying for inclusion in the review.

Results

There is evidence to support various forms of exercise including aerobic, resistance, alternative and combination exercise in the management of fatigue in early stage breast cancer patients undergoing adjuvant radiotherapy. The benefits of exercise for patients with later stage and metastatic disease is less clear and there is a lack of published research related to this category of patient.

\section{Conclusion}

Exercise is considered a safe, non-pharmacological intervention for early stage breast cancer patients receiving adjuvant radiotherapy. Further investigation is required into optimum exercise interventions and the effectiveness and viability of supervised and unsupervised models. Patient centred tailored advice and guidance needs to be developed and effectively promoted by therapeutic radiographers in order for patients to fully realise the benefit. 


\section{Background}

Breast cancer is the most common cancer diagnosed in the United Kingdom (UK). In 2015 there were 55,122 new cases of invasive breast cancer diagnosed and 11,563 breast cancer deaths (1). Cancer Research UK indicate that $81 \%$ of patients had surgery, $63 \%$ had radiotherapy and $34 \%$ had chemotherapy as part of the treatment management for breast cancer (1). Despite the proven benefits of chemotherapy, radiotherapy and hormone blocking therapy, treatment-associated fatigue is often identified as the most prevalent complaint affecting the quality of life (QoL) among breast cancer patients $(2,3)$. This fatigue is a multifaceted, subjective and physiologic state characterised by persistent, overwhelming exhaustion and decreased capacity for physical and mental work that is not relieved by rest (4). During a course of radiotherapy, treatment-related fatigue gradually increases in severity and the number of patients reporting it also increases (5). Despite this, the aetiology of fatigue is not well defined and the incidence ranges from $30-70 \%$ in women with breast cancer, reaching up to $80 \%$ when undergoing radiotherapy (6). Combined effects of treatment plus decreased levels of activity during treatment reduce the capacity for physical performance (7). This review is undertaken in order to evaluate the use of exercise in managing fatigue in breast cancer patients and to inform the therapeutic radiographer of the effectiveness of different exercise approaches and explore how optimum management of fatigue might be achieved.

\section{Methods}

A search of literature was carried out on the electronic CINAHL (Cumulative Index to Nursing and Allied Health Literature) database. The database was searched for the following terms: breast cancer, breast carcinoma, cancer related fatigue, adjuvant therapy and exercise. Full text and English language articles were cross referenced. Date limitation was applied for articles between 2005 and 2018. Potentially relevant publications were screened for retrieval. 30 potentially relevant articles were identified using the search strategy outlined in table 1 . Of these 30 articles, 6 were excluded due to non-relevance to the subject area. 24 relevant publications were used for the main analysis within this review.

\section{Discussion}

Historically, patients have been advised to avoid exercise and conserve energy during cancer treatments such as chemotherapy and radiotherapy. However, today it is widely accepted that exercise is safe and can combat fatigue, improve physical functioning and QoL (8). Aerobic exercise or cardiovascular fitness exercise aims to strengthen the heart and lungs, it raises the heart rate and enhances circulatory and respiratory efficiency. Resistance training improves muscular strength and endurance and can be achieved by movement of limbs against resistance provided by one's own body weight/gravity, elasticated bands or weights. There is still much debate regarding which type of exercise is most effective with research exploring aerobic exercise, resistance training, combinations of aerobic and resistance exercises as well as alternative exercise approaches. 


\section{Combined exercise approaches (aerobic and resistance exercises)}

The majority of research studies explore the impact of combined exercise approaches including both aerobic and resistance exercises. Mustain et al. conducted a randomised controlled trial (RCT) where participants were either assigned to a control group (with no exercise intervention) or to an exercise group (5). The exercise group were asked to increase their baseline number of steps in a day over 4 weeks and they also received a tailored resistance band exercise prescription progressing from low to moderate intensity during the trial. 38 patients completed the study and by the end of the intervention, the exercise group had increased their number of daily steps in contrast to the control nonexercise group whose daily steps had decreased from the baseline. Participants in the exercise group demonstrated improvements in reported fatigue levels from baseline to post intervention and continued improvement up to the 3-month follow-up point. The control group exhibited smaller improvement in fatigue levels from baseline to post intervention, but the fatigue levels worsened overall from the baseline to the 3-month review point. A statistical trend towards significantly lower fatigue in the exercise group than control group post intervention $(P=0.07)$ and at the 3-month follow up $(P<0.05)$ was observed (5). Results using the Functional Assessment of Chronic Illness Therapy (FACIT) subscale also supported this $(\mathrm{P}<0.05)$. This study however included both breast cancer and prostate cancer patients and therefore has not concentrated solely on the incident of breast cancer patient fatigue. The relatively small sample size is also a limiting factor however the evidence that the non-exercise group experience a reduced physical performance is also supported by the finding of Mock et al. evaluating 119 participants and concluding that treatment related toxicities in combination with physical inactivity results in a decreased physical performance (7). An aerobic exercise only trial was conducted by Mock et al. where breast cancer patients receiving adjuvant treatment were randomised into 'usual care' group or exercise group (7). The exercise entailed a brisk walk for 15 minutes increasing to 30 minutes as training progressed. Fatigue was measured in the 119 participants using the Piper fatigue scale. The programme ran for as long as the treatment regimen did. This study found a statistically significant $(\mathrm{P}=0.03)$ difference in fatigue levels after aerobic exercise using a pre-test/post-test methodology (7).

Hsieh et al. carried out a trial in 96 patients undergoing active treatment for breast cancer using an individualised whole-body approach, aerobic/resistance training (9). The sessions were individually supervised and lasted around 60 minutes, 2-3 times weekly over a period of 6 months. The Revised Piper Fatigue scale was used to measure fatigue rather than the FACIT subscale used by Mustain et al. (5). Breast cancer patients were allocated to one of four groups; patients who received surgery alone; surgery and chemotherapy; surgery and radiotherapy or the chemotherapy and radiotherapy group. The exercise intervention showed significant $(p<0.05)$ improvements in all groups in cognitive function, positive behaviour, cardiopulmonary function and experienced fatigue (9).

Campbell et al. conducted a randomised controlled pilot study with 22 breast cancer patients, also exploring the impact of a combined exercise programme, but did not find a statistically significant reduction in fatigue levels between the exercise group and control 'usual care' group during their 12-week study among patients undergoing adjuvant treatment $(p=0.115)(10)$. A trend towards lower levels of fatigue was observed but not deemed statistically significant using the Piper fatigue scale. A limitation of the study was that it only included a relatively small sample size and only included women who had early stage breast cancer. Perhaps not fully representative of the complete spectrum of patients with breast cancer. 
A review of 32 studies by Furmaniak et al. in 2016 resulted in a systemic analysis of nine studies exploring physical exercise in breast cancer patients undergoing adjuvant therapies. The review concluded that combined exercise programmes appear to slightly decrease symptoms of treatment related fatigue (11). Ligibel et al. conducted another aerobic exercise study over 12 weeks using moderate-intensity level exercise in 41 sedentary breast cancer patients (12). An activity goal of 150 minutes of aerobic exercise per week was set. Fatigue was assessed using the European Organisation for Research and Treatment of Cancer tool (EORTC). Again, there was a trend towards improved QoL with a decrease in symptoms of treatment related fatigue, although the findings were not statistically significant $(p=0.08)(12)$.

The review of the combined exercise approach provides clear support for the inclusion of exercise as a means of improving QoL and reducing treatment related fatigue. The literature reviewed identifies a range of study designs and differing fatigue measurement tools. Some have been undertaken as pilot studies or simply designed with a limited sample size in an attempt to reach a conclusion in a time limited manner. None-the-less, whether proven statistically significant or not, the general theme running through the studies is in support of the fact that the combined exercise approach, in whatever combined exercise guise is employed, is beneficial.

\section{Resistance exercise}

Resistance exercise alone has also received investigation. A randomised controlled trial by Steindorf et al. including 160 women with breast cancer, explored the impact of a progressive resistance training programme or a muscle relaxation programme, running over a period of 12 weeks (13). Fatigue was measured using the EORTC questionnaire. Overall the results demonstrated a significant decrease in fatigue in patients pre to post intervention in the exercise group, with no change in the control relaxation group (13). Schmidt et al. conducted an RCT in 101 women undergoing chemotherapy post-surgery evaluating a 12week resistance training intervention using the Fatigue Assessment Questionnaire (FAQ) (14). The findings supported that of the Steindorf et al. study (13) concluding that resistance training strongly reduces fatigue in breast cancer patients undergoing chemotherapy postsurgery.

\section{Alternative exercise approaches}

There has been a growing interest into how alternative exercise approaches, such as yoga, Nia and Qigong could be implemented to help manage fatigue amongst breast cancer patients. Lipsett et al. states that further research to determine the effect of alternative, nontraditional, modes of exercise is required (2).

An RCT of 191 breast cancer patients participating in yoga was carried out by Chandwani et al. in 2014 (15). The Brief Fatigue Inventory (BFI) was used to measure patients' fatigue levels. Patients, assigned to the yoga group or the stretching group, attended three 60minute classes per week during the six weeks of radiotherapy treatment. There was also a control 'usual care' group. Results showed significantly greater decreases in fatigue for the yoga group and the stretching group compared to the control group at the end of treatment $(p=0.04 ; p=0.02$ respectively) and marginally significant differences observed for the yoga group compared to control group at one-month post treatment $(p=0.09)(15)$. Yoga was compared to conventional exercise in another study by Lotzke et al. though the details of the 'conventional exercise programme' were limited (16). 92 women took part and no significant 
differences between the yoga group and the conventional group were observed. EORTC and Chronic Fatigue Syndrome (CFS) scales were used to measure the fatigue levels. Both interventions did not significantly improve fatigue levels (yoga $p=0.763$; conventional $\mathrm{p}=0.180)(16)$.

Nia is a non-traditional exercise type that focuses on the body, mind and spirit. It is a cardiovascular and whole-body conditioning programme. The practice of Nia can be gentle for individuals with a sedentary lifestyle or challenging for those with active lifestyles (17). 41 women took part in a study comparing Nia and 'usual care' over a 12-week period (17). The Nia group practised Nia for 20-60 minutes three times a week. The 'usual care' group were asked to continue normal daily activities. Results demonstrated that the Nia group reported significantly less fatigue between week 6 and 12 than the usual care group $(p=0.05)(17)$. However, the study's sample size limits the power of the study and it is reported that the age of the Nia group averaged five years younger than the control group - it is possible that age might be linked to physical ability and this could have influenced results.

Qigong is an ancient Chinese practice of mind-body intervention (18). A study comparing Qigong to usual care was carried out in 96 women undergoing radiotherapy for breast cancer (18). The Qigong group were asked to carry out the practice 5 times a week for 40 minutes per session. Fatigue was assessed using the Brief Fatigue Inventory (BFI). Results demonstrated reduced fatigue in women who had expressed higher levels of depressive symptoms that were assigned to the Qigong group rather than the control group $(p<0.1)$ (18). This practice may require more investigation as it is showing benefit in relation to fatigue in only a limited sub-group of breast cancer patients.

\section{Impact of unsupervised versus supervised exercise programmes}

Another aspect for consideration regarding the impact of exercise on fatigue, is whether supervised or unsupervised/home-based programmes are more effective. Lipsett et al. carried out a systematic review and meta-analysis of exercise on fatigue in breast cancer patients (2). They included nine studies and found statistically significant benefits of supervised, combined aerobic and resistance training. Van Vulpen et al.'s similar metaanalysis also supports this theory finding that the trials that analysed supervised exercise programmes showed slightly greater impact on general and physical dimensions of fatigue (3). It could be argued that supervised exercise trials appear more effective because patients adhere better to the programme as they are more accountable as their attendance at sessions will be noted and more encouragement can be given to maintain activity levels (19). In contrast the homebased/unsupervised studies are more likely to have lower compliance rates as it is up to the patient to comply and will require more self-motivation. Harte states that exercise can pose a significant challenge for people who are feeling physically and emotionally drained (8). Unsupervised exercise programmes may be more flexible for certain groups of patients where commitments such as work, or child care obligations make it more difficult to attend set sessions with a trainer or a gym. Well adhered to home-based programmes, with effective support and compliance monitoring may be the preferred option in some cases. The wearing of electronic exercise monitors or wrist bands is one method of compliance monitoring or motivation that might be explored.

The simple idea of providing information and educating patients on the benefits of physical activity during their treatment is something that has received some attention. A study by Windor et al. explored the idea of providing information at the start of treatment on exercise and fatigue (20). Patient fatigue levels were measured using the BFI. They were given a 
folder with information on how to manage fatigue with exercise and information on workshops that would be run locally that they could attend if they desired. Results demonstrated that $70-78 \%$ of patients found the information provided useful or very useful with $70.2 \%$ reporting exercising during their treatment. This supports the argument that providing the information to patients in some cases can be enough and allows the patient the freedom to participate as much or as little as they desire or indeed can manage. However, the results of the study also showed that the patients who reported using the information also reported experiencing higher levels of fatigue. This might be attributed to attempting more physical exercise than perhaps the patient life-style, even pre-diagnosis, was used to performing. Attempting to introduce exercise over and above the norm and not being able to sustain the exercise regime can trigger an understandable human response to attribute the failure to perform on fatigue. The patients after all are being conditioned to expect fatigue as

a result of their treatment! Perhaps patients who were experiencing heightened fatigue levels decided to use the information that had been provided. The reporting of the information being useful coming to light as a result of experiencing higher fatigue levels. A limitation of the study is that the exercises varied considerably from a short walk to more intensive scheduled high intensity workouts. The targeting of individual information to specific patients using smart phone applications or similar media is something that might be considered for the future. Mobile applications might also serve as the mechanism for exercise compliance monitoring.

It can also be argued that supervised group exercise programmes may simply have a psychological advantage and that exercising as part of a group rather than alone can influence a patient's perspective of fatigue especially given the self-reporting data collection tools. However, the study by Steindorf et al. does not support this theory as the study ran a control supervised relaxation group alongside the supervised exercise group (13). Their results showed that the exercise group had decreased levels of fatigue but the control group, of supervised relaxation, did not observe this decrease in fatigue. This further supports the argument that exercise must provide beneficial effects beyond psychosocial effects induced by group-based programs (13). Schmidt et al. had similar findings in the supervised resistance training programme of 101 patients over a 12-week period (14). In week 13 fatigue levels had increased in the control group of supervised relaxation class while in the exercise group they did not display an increase.

\section{Impact of intensity and frequency of exercise}

Another dimension of exercise that needs to be considered alongside the type of exercise and how it is delivered, is the intensity and frequency of exercise required to provide optimal management of fatigue. A meta-analysis into the optimal level of exercise required was carried out by Carayol et al. in 2013 (21). Results from the 17 combined studies reviewed showed that the most significant reduction in fatigue occurred with moderate intensity exercise. Relatively low levels of moderate intensity exercise appear to be most effective in decreasing fatigue $(21,22)$. Hseih et al.'s clinical trial also supports the notion that moderate intensity exercise is effective against fatigue during adjuvant therapy (9). However, they do acknowledge that more research is needed into higher level intensity exercise to establish if this is safe or any more effective in comparison to moderate intensity. 


\section{Advancing future professional practice}

Further investigation and extensive studies in the area would be beneficial to establish that exercise is effective and safe for late stage patients and for patients with metastatic disease. It has been suggested in the literature that the less well known, alternative exercise approaches, could be beneficial for the less agile and less mobile patient $(17,18)$. Perhaps the alternative exercise approaches may also be more applicable for use with patients with late stage and metastatic disease. Additionally, there are challenges in terms of patient compliance with exercise advice as patients face a range of barriers including, most predominantly, fatigue itself, followed by pain, difficulty getting motivated and difficulty remaining disciplined $(8,23)$. Effective, targeted, education for the patient and appropriate information provision for fatigue management interventions is imperative.

Exercise can be easily implemented and can be a low-cost initiative to manage fatigue $(5,7,24)$. There is some evidence to suggest that exercise can favourably modify hormones, growth factors, adipokines and other cytokine biomarkers and thus in turn improve fatigue levels (7). Groeneveld et al. found that physical exercise had likely contributed to the patient's ability to return to work, primarily by increasing energy levels (25). Decreasing the level of fatigue improves physical functioning and facilitates return to work and can serve to improve patient self-esteem and wellbeing. In the future, more extensive larger scale studies would be beneficial to help establish which type of exercise is most effective as some of the studies have been inconclusive due to relatively small participant numbers and the lack of robust control groups (26).

\section{Conclusion}

The majority of literature reviewed focuses on early stage breast cancer patients with late stage or patients with metastatic disease receiving little focus in terms of exploring the beneficial impact that exercise has on treatment related fatigue. Perhaps this is not surprising as from corporate memory, therapeutic radiographers, health professionals and clinical oncologists would not have proactively promoted exercise in the past as an intervention to manage treatment related fatigue. Modern day approaches certainly advocate exercise interventions and conservatively, its adoption and effective introduction by therapeutic radiographers and other health care professionals will take time to inculcate into practice and disseminate throughout the radiotherapy profession. Many may continue to consider that advocating exercise regimes at a time when patients are still coming to terms with their diagnosis and comprehending treatment, is a low priority. The evidence is clear that exercise can benefit patients, it requires professional judgement in its prescription and application and to ensure that patients fully comprehend the expectation in order to gain benefit. More investigations focusing on patients with late stage and metastatic disease would help clarify if exercise is effective or indeed safe for that patient group.

Fatigue is multidimensional in nature and its reporting is subjective and open to bias. This makes fatigue difficult to measure and a notable limitation in terms of reporting fatigue in research is the range of tools that are used to measure fatigue dimensions including the Piper Fatigue Scale, Multidimensional Fatigue Inventory (MFI), Brief Fatigue Inventory (BFI), Fatigue Assessment Questionnaire (FAQ) and Functional Assessment of Chronic Illness Therapy subscale (FACIT). It can be challenging when attempting to compare the results of one trial to another and to test the validity of one tool against another. An extensive study to identify and robustly validate an effective tool for gauging fatigue would be useful. 
Supervised and unsupervised home-based programmes both showed promise but there is a lack of definitive evidence as to which is most effective as few studies directly compared them against each other. Individualised plans may in fact be the ideal as this allows exercise to be tailored specifically to the patient. The challenge is that every patient has a unique situation and circumstances so supervised exercise might be best for those requiring more support. More independent individuals or those with work or other life commitments may benefit from homebased-unsupervised programmes. Both styles have their place depending on the individual patient needs.

There is substantial evidence in literature to indicate that exercise, in numerous forms, can help manage fatigue in breast cancer patients undergoing adjuvant radiotherapy. Arguments have been made in favour of all exercise approaches - aerobic, resistance, combination and alternative. They have been investigated against control groups but there is a lack of studies to compare the approaches against each other. There is a growing base of thought towards using a combination of traditional exercise forms alongside alternative approaches such as yoga and Nia to optimum effect. For the future, more extensive, larger scale studies would be beneficial to help establish unequivocal evidence of the benefits of particular, clearly defined, exercise approaches in managing fatigue related symptoms among breast cancer patients undergoing adjuvant radiotherapy.

Exercise is considered a safe, non-pharmacological intervention for managing fatigue experienced by early stage breast cancer patients receiving adjuvant radiotherapy; but there is a definite gap in research regarding the use of exercise in later stage and patients with metastatic breast cancer. Moderate intensity exercise regimes have received most consideration and further investigation is required into optimum exercise interventions and the effectiveness and viability of supervised and unsupervised models. Exercise is an effective, self- help tool that health care professionals are often poor at promoting. Patient centred, tailored, advice and guidance needs to be developed and effectively promoted by therapeutic radiographers in order for patients to fully realise the benefit.

Acknowledgements. The authors thank the staff of the radiotherapy department, Western Health and Social Care Trust, for their support.

Financial Support. This review received no specific grant from any funding agency, commercial or not-for-profit sectors.

Conflicts of interest. None. 
Table 1: CINAHL (Cumulative Index to Nursing and Allied Health Literature) database. Search Strategy.

\begin{tabular}{|c|c|c|c|c|}
\hline $\begin{array}{l}\text { Search } \\
\text { ID \# }\end{array}$ & Search Terms & Search Options & Last Run Via & Results \\
\hline S6 & $\begin{array}{l}\text { (S1 AND S2) } \\
\text { AND adjuvant } \\
\text { therapy }\end{array}$ & $\begin{array}{l}\text { Limiters - Date Published: } \\
\text { 20060101-20171231 } \\
\text { Expanders - Apply equivalent } \\
\text { subjects } \\
\text { Search modes - Boolean/Phrase }\end{array}$ & $\begin{array}{l}\text { Interface - EBSCOhost } \\
\text { Research Databases } \\
\text { Search Screen - Basic } \\
\text { Search } \\
\text { Database - CINAHL } \\
\text { Complete }\end{array}$ & 30 \\
\hline S5 & $\begin{array}{l}\text { (S1 AND S2) } \\
\text { AND adjuvant } \\
\text { therapy }\end{array}$ & $\begin{array}{l}\text { Limiters - Date Published: } \\
20060101-20171231 \\
\text { Expanders - Apply equivalent } \\
\text { subjects } \\
\text { Search modes - Find all my search } \\
\text { terms }\end{array}$ & $\begin{array}{l}\text { Interface - EBSCOhost } \\
\text { Research Databases } \\
\text { Search Screen - Basic } \\
\text { Search } \\
\text { Database - CINAHL } \\
\text { Complete }\end{array}$ & 57 \\
\hline S4 & $\begin{array}{l}\text { (S1 AND S2) } \\
\text { AND adjuvant } \\
\text { therapy }\end{array}$ & $\begin{array}{l}\text { Expanders - Apply equivalent } \\
\text { subjects } \\
\text { Search modes - Find all my search } \\
\text { terms }\end{array}$ & $\begin{array}{l}\text { Interface - EBSCOhost } \\
\text { Research Databases } \\
\text { Search Screen - Basic } \\
\text { Search } \\
\text { Database - CINAHL } \\
\text { Complete }\end{array}$ & 63 \\
\hline S3 & S1 AND S2 & $\begin{array}{l}\text { Expanders - Apply equivalent } \\
\text { subjects } \\
\text { Search modes - Find all my search } \\
\text { terms }\end{array}$ & $\begin{array}{l}\text { Interface - EBSCOhost } \\
\text { Research Databases } \\
\text { Search Screen - Basic } \\
\text { Search } \\
\text { Database - CINAHL } \\
\text { Complete } \\
\text { CINAHL Complete }\end{array}$ & 344 \\
\hline S2 & $\begin{array}{l}\text { Cancer related } \\
\text { fatigue AND } \\
\text { exercise }\end{array}$ & $\begin{array}{l}\text { Limiters - Available in Library } \\
\text { Collection } \\
\text { Expanders - Apply equivalent } \\
\text { subjects } \\
\text { Search modes - Find all my search } \\
\text { terms }\end{array}$ & $\begin{array}{l}\text { Interface - EBSCOhost } \\
\text { Research Databases } \\
\text { Search Screen - Basic } \\
\text { Search } \\
\text { Database - CINAHL } \\
\text { Complete }\end{array}$ & 926 \\
\hline S1 & $\begin{array}{l}\text { Breast cancer } \\
\text { OR breast } \\
\text { carcinoma }\end{array}$ & $\begin{array}{l}\text { Limiters - Available in Library } \\
\text { Collection } \\
\text { Expanders - Apply equivalent } \\
\text { subjects } \\
\text { Search modes - Find all my search } \\
\text { terms }\end{array}$ & $\begin{array}{l}\text { Interface - EBSCOhost } \\
\text { Research Databases } \\
\text { Search Screen - Basic } \\
\text { Search } \\
\text { Database - CINAHL } \\
\text { Complete }\end{array}$ & 318,393 \\
\hline
\end{tabular}




\section{References.}

1. Cancer research UK. Breast Cancer Statistics. 2015.

http://www.cancerresearchuk.org/health-professional/cancer-statistics/statistics-bycancer-type/breast-cancer Accessed on 21st October 2018.

2. Lipsett A, Barrett S, Haruna F, Mustain K, O'Donovan A. The impact of exercise during adjuvant radiotherapy for breast cancer on fatigue and quality of life: $\mathrm{A}$ systematic review and meta-analysis. The breast. 2017; 32:144-155.

3. Van Vulpen JK, Peeters PHM, Velthuis MJ, Van der Wall E, May AM. Effects of physical exercise during adjuvant breast cancer treatment on physical and psychosocial dimensions of cancer-related fatigue: A meta-analysis. Maturitas. 2016; 85:104-111.

4. Berger AM, Gerber L, Mayer DK. Cancer related fatigue - implications for breast cancer survivors. Cancer. 2012; 118(58): 2261-2269.

5. Mustain K, Peppone L, Darling TV, Palesh O, Heckler CE, Morrow GR. A 4-week home based aerobic and resistance exercise programme during radiation therapy: $\mathrm{A}$ pilot randomized clinical trial. Journal Support Oncology. 2009; 7:158-167.

6. Alcantara-Silva TR, Freitas-Junior R, Freitas N, Machado G. Fatigue related to radiotherapy for breast and/or gynaecological cancer. Journal of clinical nursing. 2013; 22: 2679-2686.

7. Mock V, Frangakis C, Davidson NE et al. Exercise manages fatigue during breast cancer treatment: a randomised control trial. Psycho-oncology. 2005; 14: 464-477.

8. Harte J. Exercise during and after breast cancer treatment, Alternative and complimentary therapies 2014; 20: 259-262.

9. Hsieh C, Sprod L, Hydock D, Carter S, Hayward D, Schneider CM. Effects of a supervised exercise intervention on recovery from treatment regimens in breast cancer survivors. Oncol Nurs Forum. 2008; 35: 909-915. 
10. Campbell A, Mutrie N, White F, McGuire F, Kearney N. A pilot study of a supervised exercise programme as a rehabilitation treatment for women with breast cancer receiving adjuvant treatment. European Journal of Oncology Nursing. 2005; 9: 56-63.

11. Furmaniak AC, Menig M, Markes MH. Exercise for women receiving adjuvant therapy for breast cancer (review), Cochrane database for systematic reviews. 2016; 9 : DOI10.1002/14651858.CD005001.pub3.

12. Ligibel JA, Partridge A, Giobbie-Hurder A et al. Physical and psychological outcomes among women in a telephone-based exercise intervention during adjuvant therapy for early stage breast cancer. J Women's Health. 2010; 19: 1553-1559.

13. Steindorf K, Schmidt ME, Klassen O et al. Randomized controlled trial of resistance training in breast cancer patients receiving adjuvant therapy: results on cancerrelated fatigue and quality of life. Ann of Oncol. 2014; $25: 2237-2243$.

14. Schmidt ME, Wiskemann J, Armbrust P, Schneeweiss A, Ulrich C, Steindorf K. Effects of resistance exercise on fatigue and quality of life in breast cancer patients undergoing adjuvant chemotherapy: A randomized control trial. Cancer Therapy. 2015; 137: 471-480.

15. Chandwani KD, Perkins G, Nagendra HR et al. Randomized, controlled trial of yoga in women with breast cancer undergoing radiotherapy. Clin Oncol. 2014; 32:10581065.

16. Lotzke D, Wiedemann F, Recchia D et al. lyengar-Yoga compared to exercise as a therapeutic intervention during (neo) adjuvant therapy in women with stage I-III breast cancer. Evidence-Based Complementary and Alternative Medicine. 2016; 2016: 1-8.

17. Reis D, Walsh E, Young-McCaughan S, Jones T, Effects of Nia exercise in women receiving radiation therapy for breast cancer. Oncol Nurs Forum. 2013; 40: 374-382.

18. Chen Z, Meng Z, Milbury K et al. Qigong improves the quality of life in women undergoing radiotherapy for breast cancer. American Cancer Society. 2013; 119: 1690-1698. 
19. Meneses-Echavez JF, Gonzalez-Jimenez E, Ramirez-Velez R. Supervised exercise reduces cancer-related fatigue: a systematic review. Journal of Physiotherapy. 2015; 61: 3-9.

20. Windor PM, Potter J, McAdam K, McCowan C. Evaluation of a fatigue initiative: Information on exercise for patients receiving cancer treatment. Clin Oncol. 2009; 21: 473-482.

21. Carayol M, Bernard P, Boiche J et al. Psycological effect of exercise in women with breast cancer receiving adjuvant therapy: what is optimal dose needed. Ann of Oncol. 2013; 24: 291-300.

22. Carayol M, Delpierre C, Bernard P, Ninot G. Population-,intervention- and methodology related characteristics of clinical trials impact exercise efficacy during adjuvant therapy for breast cancer: a meta-regression analysis. Psycooncology. 2015; 24: 737-747.

23. Romero S, Li Q, Mao JJ. Factors and barriers associated with changes in physical activity after cancer diagnosis. Journal of Clinical Oncology. 2017; 35(5) 162.

24. Schmitz K, DiSipio T, Gordon L, Hayes S, Adverse breast cancer treatment effects: the economic case for making rehabilitative programmes standard of care. Support care cancer. 2015; 23: 1807-1817.

25. Groeneveld IF, De Boer AG, Frings-Dresen MH. Physical exercise and return to work. J cancer surviv. 2013; 7: 237-246.

26. Abed J, Dolan L, Jones J, Dinniwell R. Impact of exercise on reported fatigue and anxiety levels in early stage breast cancer undergoing radiotherapy. Journal of medical imaging and radiation sciences. 2017; 48: S5 